\title{
Spatial memory training in a citizen science context
}

\begin{abstract}
Memory deficit is one of the primary effects of intellectual disability, and has a great impact on daily life. Here, we propose a novel spatial memory training system based on a citizen science virtual environment, in which users navigate an aquatic robot in a polluted canal and identify specific objects from images acquired by the robot. A portable low-cost electroencephalography device is utilized to enhance the degree of interactivity and enable real-time estimation of the affective state of the user. We involved a cohort of 60 healthy adult subjects to evaluate users' interest, memory performance, and affective variables as a function of navigation modality (active versus passive) and interface (a traditional computer mouse versus the headset). Despite offering a higher level of difficulty, the headset was preferred over a traditional mouse control by the users, whose spatial memory performance did not vary with the navigation modality or the interface. Active navigation was found to lead to a higher level of engagement, as measured by the headset. These findings suggest the possibility of a new, effective, and entertaining form of intellectual rehabilitation with potential impact on fetal alcohol syndrome.
\end{abstract}

Keywords: Affective state evaluation, Citizen science, Fetal alcohol syndrome, Intellectual disability, Spatial memory training.

\section{Introduction}

Memory deficit is one of the primary effects of intellectual disability, and highly impacts daily life [30]. Among the different causes of intellectual disability, fetal alcohol syndrome (FAS) is considered the most relevant by incidence in the United States and Western Europe [1]. Such a syndrome is the combination of morphological, neurological, and intellectual impairments, and its etiology is related to alcohol consumption during pregnancy [83]. Exposure of the fetus to alcohol during the first weeks of gestation affects brain cell replication and migration, resulting in brain malformation [18]. The clinical picture of patients with FAS includes problems in executive functions, working memory, cognitive skills, and verbal fluency, resulting into a general learning deficit accompanied by attention deficit and hyperactivity disorder (ADHD) [28, 9].

Deficits in executive functions and working memory are mainly associated with malformations of the frontal lobe of the brain [69]. These impairments impinge on academic performance and often result in scarce autonomy in daily life $[14,3]$. Prenatal alcohol exposure has been shown to also cause defects in other brain areas, such as the hippocampus [8], which is considered to host a type of long-term memory important for orientation and spatial navigation [59, 11]. Related intellectual impairments have been identified in pre-clinical studies on animal models [25] via the Morris maze task [55].

A range of possibilities to train people in cognitive recovery has been explored over the years [39]. Training strategies based on rehearsal $[45,32]$ have shown a positive effect not only on working memory $[19,4]$, but also on long-term memory capabilities [32]. In addition, computer-based gaming systems have been proposed as viable training strategies for visual working memory insufficiency $[37,5,10,79]$, which is a specific, yet common, impairment in FAS patients.
In this context, virtual versions of the Morris maze task have been designed for training and testing human spatial memory [72]. The virtual reality environment could be an exact representation of a Morris maze tank [66, 31, 78], or a representation of a more familiar and stimulating environment for human subjects [47, 71]. For example, in [71], a virtual environment reproducing a bungalow with different rooms to be explored was used to test spatial memory in patients with brain injury. Results in [71] confirm that patients present reduced spatial memory capabilities and highlight the positive effect of active navigation in the test. Specifically, patients were found to be more successful in the location of spatial layouts encountered during the trip through the rooms when taking an active role in exploring the environment through a computer interface.

Here, we demonstrate a novel system for intellectual rehabilitation, which leverages untapped motivational aspects associated with computer-based citizen science. Citizen science refers to participation of volunteers in research projects led by professional scientists $[77,20]$. This form of crowd science has been recently gaining traction as a way to collect and analyze large datasets where analysis by technical experts or dedicated computer programs is practically and economically unfeasible [29]. A wide range of motivational drivers influences the contribution of volunteers to citizen science projects, including individual interest in the subject of the project and excitement that they experience in contributing to research $[57,73,65,16]$. We recently demonstrated $[17,42,60]$ that the field of rehabilitation can benefit from the motivational incentives afforded by citizen science, whereby citizen science activities can be effectively integrated in a computer-mediated physical rehabilitation. Specifically, subjects undergoing rehabilitation treatments in which their physical activity contributes to a citizen science project or, simply, some form of science learning show improved adherence to rehabilitation regimens and a better per- 
formance in motor tasks [42].

The outcomes of this study are envisaged for future use on FAS patients and is designed around a successful environmental citizen science project (Brooklyn Atlantis: brooklynatlantis.com). The project is aimed at monitoring the pollution level of a Superfund site, the Gowanus canal in Brooklyn, NY, by uploading pictures and water quality data collected by an aquatic robot periodically deployed in the body of water. These data are then assigned to volunteers for classification from their homes through the internet. We expect that the possibility of virtually navigating the canal and locating specific objects could constitute a valid training activity for both visuo-spatial working memory and long-term memory. In addition, we hypothesize that the interest in this highly technological, yet tangible, project together with gamification instances [13, 43] could compensate for the lack in attention associated with the syndrome and enhance the effectiveness of the rehabilitation activity.

The reduced capability of FAS patients to concentrate on an intellectual task could lead to the failure of training programs [70]. A relatively new approach to cope with the lack of attention during intellectual training is the introduction of bio-feedback. Such a methodology integrates different sensors that can measure the subject's level of engagement, such as Galvanic skin responses $[54,81]$ or electroencephalography (EEG) signals $[15,12,87]$, to create an acoustic or visual feedback [40], or adjust game difficulty [48]. The result is a patientspecific rehabilitation system that adapts itself based on realtime measurement of the subject's engagement.

Based on these promising results, we integrate our new system with an off-the-shelf device for bio-feedback, which enables real-time data processing of the user EEG signals, the Emotiv EPOC headset [23]. Among other functionalities, such as facial expression recognition and trainable thought recognition [2], the device is able to run embedded algorithms for user emotion estimation, so-called "affective" state [84]. From EEG signals acquired on the scalp, the headset extracts a combination of features to provide a real-time assessment of a set of affective variables named as: "Long term Excitement", "Short term Excitement", "Meditation", "Frustration", and "Engagement" [53], otherwise obtainable via more complex EEG-based laboratory setups [34]. While the quality of the raw voltage data acquired by the EPOC has been deemed acceptable for nonclinical applications [22], no validation of the affective variables estimation has been performed thus far $[80,86,76]$.

The objective of this study is threefold. We first verify the feasibility of memory training through a citizen science activity on a group of healthy adult subjects. In particular, this effort seeks to assess if a spatial memory task, in the form of a virtual Morris maze in a citizen science context, is perceived by the participants as engaging and interesting. Second, this study explores whether spatial memory performance in participants can be enhanced through active navigation of the environment using the EPOC headset. Finally, this effort evaluates the possibility of integrating the estimation of the subjects' affective state through the EPOC headset toward future applications in bio-feedback.

\section{Materials and methods}

\subsection{Experimental setup}

The experimental set-up consists of a laptop computer and the Emotiv EPOC headset (Emotiv Inc., San Francisco, CA, USA), shown in Figure 1-(a). The EPOC headset is a low-cost EEG device for human-computer interface, with 16 electrodes spatially distributed following the 10-20 standard [33]. Beyond acquiring the raw voltage signal from the electrodes, the device evaluates the five affective variables ("Long term Excitement", "Short term Excitement", "Frustration", "Meditation", and "Engagement"), each receiving a value ranging from 0 to 1. This lightweight device is connected via Bluetooth to a computer, permitting to the user to freely move their head. A dualaxis gyroscope sensor is embedded in the device, offering an additional input source for the analysis.

We developed the software using the National Instruments LabVIEW programming environment (release 12.0, NI, Austin, TX, USA) with the aims of: (i) allowing the user to explore the Brooklyn Atlantis panoramic pictures and virtually navigate the canal; (ii) performing a memory test after the navigation task; (iii) using the gyroscope signals from the headset to control the navigation; and (iv) acquiring and logging affective variables. The interfacing of the device in the LabVIEW environment is realized using the LabVIEW Emotiv toolkit [56]. The voltage signals are acquired by the device at a sampling rate of $128 \mathrm{~Hz}$. The affective variable emission is event-triggered. A new data bundle is emitted when the onboard algorithm recognizes a socalled affective event.

The spatial memory trial is articulated in three phases. During the first phase (also referred to as the navigation phase), the subject is provided with a virtual reality environment, shown in Figure 1-(b). The environment is represented by a spherical panoramic picture taken in the Gowanus canal. A subject can explore the spherical pictures by controlling the view angle in terms of heading (horizontal) and pitch (vertical) rotations. In addition, the subject is presented with a second picture on the interface, reproducing the map of the canal. A red dot on the map represents the spot where the current panoramic picture was taken and a circular sector indicates the current view angle, Figure 1-(b). The topographic images in the background in the navigation map are uploaded from Google Maps [49].

The spherical pictures can be explored with two different interfaces: a mouse (M) and the headset $(\mathrm{H})$. In the former case, the subject uses a traditional computer mouse to move two pointer sliders of the interface, one for each angle, see Figure 1-(b). In the latter case, the panoramic picture is explored through head movements, tracked by the gyroscope embedded in the headset. The vertical view angle is proportional to the pitch angle of the head, obtained by integrating one of the gyro signals. By turning their head to the left or to the right, the subject scrolls the view in the respective direction. For such a movement, the horizontal scrolling velocity is proportional to the heading angle of the head obtained by post-processing the second gyro signal. A "Gyro Reset" button on the interface allows the user to reset the neutral position of the head with respect to the screen. 


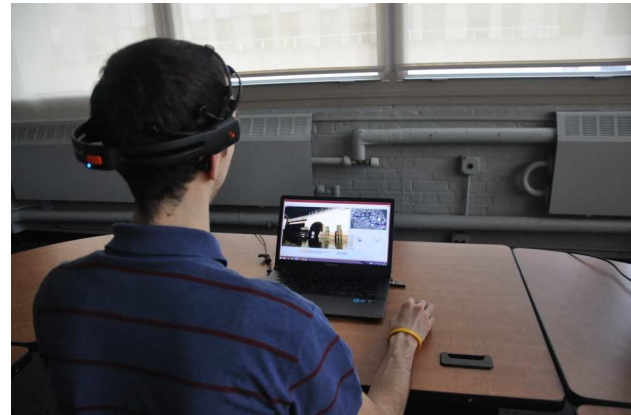

(a)

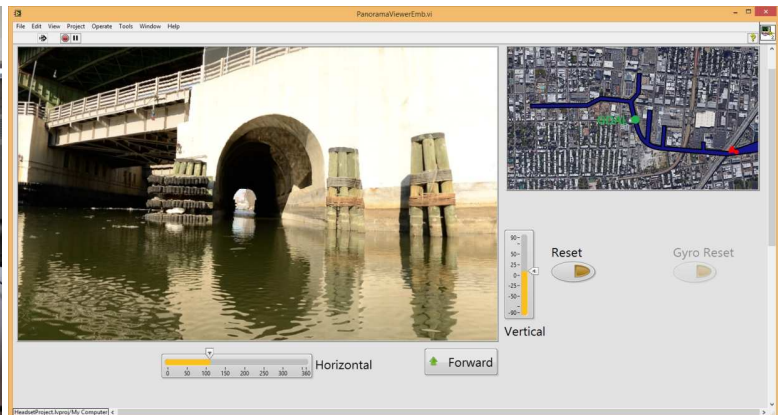

(b)

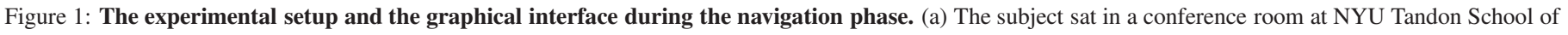

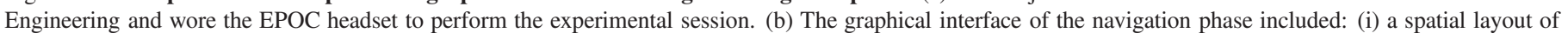

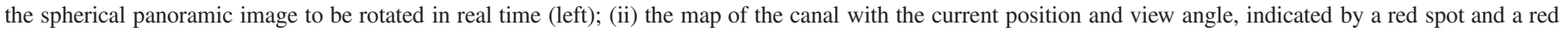
circular sector, respectively (top-right); and (iii) a set of control inputs, including two pointer sliders and buttons (bottom).

We considered two different modalities for the navigation phase: active $(\mathrm{A})$ and passive $(\mathrm{P})$. During active navigation, when the panoramic image is rotated so that the subject is facing one of the possible directions of progression, a "Forward" button is enabled. By clicking the button the subject moves in the selected direction and the interface is updated with the closest panoramic image available for that direction. Conversely, when the panoramic image is rotated so that it faces a non-allowed direction of progression (such as the edge of the canal for example), the "Forward" button is disabled. Subjects are asked to reach the target point indicated as the goal on the map by moving progressively from one spot to another, see Figure 2(a). They are free to take as much time as they want to reach the goal and freely move in any direction allowed, with no limit on the distance from the goal point, but they are explicitly recommended to explore and memorize the environment and the current position on the map before leaving one spot. During passive navigation, the image is updated automatically by the software after a predefined time. In such a time interval, subjects could explore the panoramic picture autonomously. No goal point is shown on the map and free movements are not allowed along any direction.

During the second phase (also referred to as the recalling phase) the subject is asked to recognize the location of objects in the explored environment. A pop-up picture of an encountered spatial layout is shown to the participant for three seconds. Then, the pop-up picture is closed and a wider version of the canal map with a few superimposed clickable spots is presented, as shown in Figure 2-(b). The subject is tasked with clicking the spot on the map where they recall the particular object was located. After the click, one of the two indicators on the interface lights up to show to the subject if their answer is correct or incorrect, as shown in Figure 2-(b). The sequence of picture to recall and map with clickable spots is repeatedly presented for a total of six times, with a specific unique object presented in each picture. For each correctly located picture, the subject gains a score of 50 points for a maximum of 300 . There is no penalty for incorrect answers. At the end of the sequence, the overall quantitative score is displayed. This is the third phase of the trial (also referred to as score viewing phase), as shown in Figure 2-(c).

\subsection{Experimental protocol}

A cohort of 60 normally developed adults, 49 males and 11 females, aged $25.2 \pm 4.5$ year, were enrolled in the study. The experiment, along with the consent procedure, was approved by the NYU institutional review board (IRB\# 14-10174). Subjects were recruited among staff, students, and faculty of the NYU Tandon School of Engineering. If their consent to participate in the study was obtained, they were directed to a conference room hosting the setup, see Figure 1-(a). All the subjects were tested individually. Each subject was asked by an experimenter from our group to wear the headset, following the manufacturer's instructions. The Emotiv Control Panel software was used to ascertain the quality of the EEG signals before starting the trials. Participants received background information about the Brooklyn Atlantis project and the goal of their virtual navigation in the Gowanus canal as a citizen science activity for environmental monitoring. They were encouraged to pay attention as much as possible to the scenes encountered during their navigation in a memory test.

Four different experimental conditions were considered in this study: AH, AM, PH and PM. Each subject performed four consecutive trials in which we varied the interface $(\mathrm{M}$ or $\mathrm{H})$ and navigation modality (A or $\mathrm{P}$ ). To compensate for learning effects, the software enabled virtual navigation along two different paths in the canal, both paths having the same ending point, approximately located in the middle of the canal, but starting from two opposite ends. We refer to these two starting points as north (N) and south (S). The subject always started with an active trial from one of the two possible starting points. During active navigation, the amount of time that the subject required to reach the goal point was recorded. Then, the subject performed the recalling phase and saw the relative score obtained for the memory test.

After completing this trial, the subject performed a second trial, consisting of passive navigation, using the same interface, but starting from the opposite end of the canal. As mentioned, 


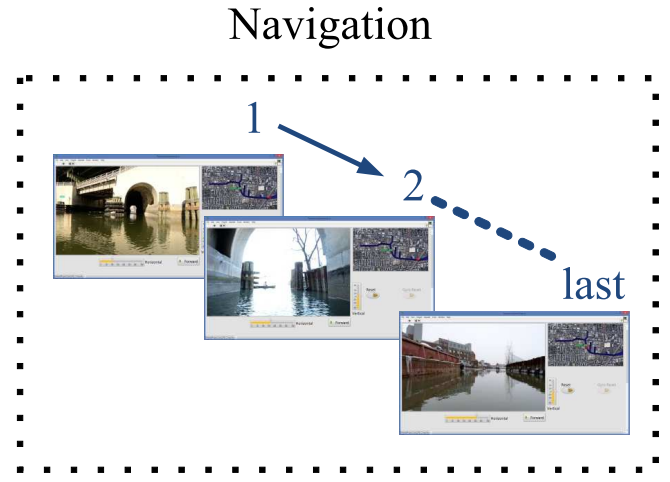

(a)

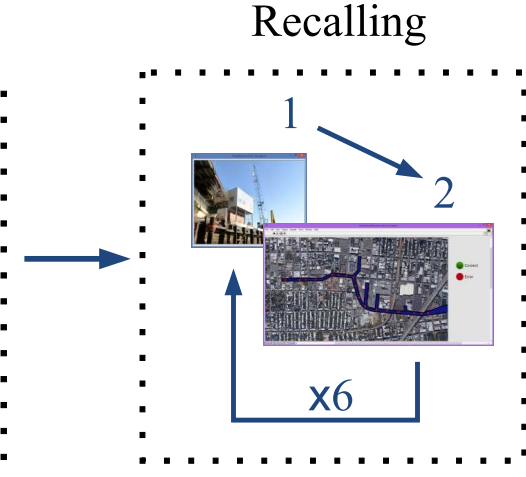

(b)
Score viewing

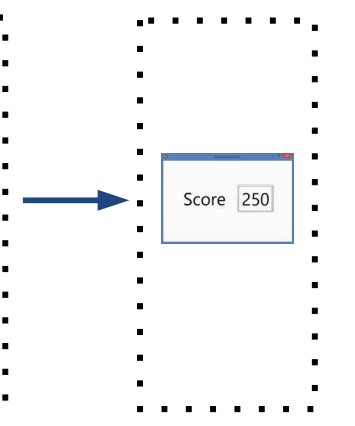

(c)

Figure 2: The graphical interface presented to participants during a trial. During the navigation phase (a), the scrolling panoramic image and the spot indicating the current position on the map change according to the virtual navigation from the first spot to the goal ("last"). During active navigation, the subject determines both the sequence of the positions along the canal and the time spent on each of them. During passive navigation, the sequence of positions and the corresponding time spent are automatically imposed. During the recalling phase (b), an image showing an object encountered during navigation is presented for three seconds, followed by a map with clickable spots superimposed to the traversed positions. The indicators on the right side of the map window indicate a correct (green light) or incorrect (red light) answer. Such a sequence in the recalling phase is repeated six times. In the score viewing phase (c), the total score is shown to the subject.

the software imposed a total duration for the passive trial equal to the time spent by the subject to reach the goal in the previous active trial. The subject could explore each panoramic picture before been moved to the next one. The total time was equally partitioned for each panoramic picture. Upon completing the corresponding recalling and score viewing phases, the subjects performed a third trial, which consisted of an active trial starting from the opposite end, with respect to the first trial, and using the alternative interface ( $\mathrm{H}$ instead of $\mathrm{M}$ or vice versa). In the fourth and last trial, the subject used the same interface used in the third trial for passive navigation, starting from the opposite end of the canal. During the four trials, subjects traversed the two paths twice ( $\mathrm{N}$ and $\mathrm{S}$ ) in different conditions, but in the recalling phase delivered in the second and fourth trials, pictures from four different sets were presented to them. We assigned subjects to four different groups, as shown in Table 1.

Table 1: Order of performance of the four trials. $\mathrm{A}=$ Active navigation modality, $\mathrm{P}=\mathrm{Passive}$ navigation modality, $\mathrm{M}=$ Computer mouse interface, $\mathrm{H}=$ Headset interface, $\mathrm{N}=$ North end starting path, $\mathrm{S}=$ South end starting path. As an example, an AH-N trial means that the subject performed a trial in active navigation, using the headset, and starting from the north end of the canal.

\begin{tabular}{ccccc}
\hline Trial 1 & Trial 2 & Trial 3 & Trial 4 & Subjects \\
\hline AH-N & PH-S & AM-S & PM-S & 15 \\
AM-N & PM-S & AH-S & PH-N & 15 \\
AH-S & PH-N & AM-N & PM-S & 15 \\
AM-S & PM-N & AH-N & PH-S & 15 \\
\hline
\end{tabular}

At the end of the trials, each participant was asked to fill out a survey. The survey consisted of nine items (Q1-Q9). The first two items asked the subjects their gender and if they were registered users on the Brooklyn Atlantis website [58]. The remaining items are reported below. For each item, except Q9, the subjects chose a level of agreement using a five point Likert scale (strongly agree, somewhat agree, neutral, somewhat disagree, and strongly disagree). Possible answers for Q9 instead, were: "Mouse controlled" and "Head controlled".

Q.3 The 3D navigation memory test (mouse controlled) was interesting.

Q.4 The 3D navigation memory test (mouse controlled) was fun.

Q.5 The 3D navigation memory test (mouse controlled) was difficult.

Q.6 The 3D navigation memory test (head controlled) was interesting.

Q.7 The 3D navigation memory test (head controlled) was fun.

Q.8 The 3D navigation memory test (head controlled) was difficult.

Q.9 Which task would you like to repeat?

\subsection{Data processing and statistical analysis}

Responses to survey questions Q3-Q8 were associated with a score, ranging from 1 to 5 , increasing with the level of agreement. First, significant differences in the answers' distribution, with respect to random chance, were evaluated using the chisquare test [62]. Then, survey answers were paired depending on the specific aspect they were intended to address, when using the mouse and the headset, respectively. Specifically, Q3 and Q6 were intended to address the interest in the activity, Q4 and Q7 the fun, and Q5 and Q8 the difficulty. Significant differences between average values for each pair were assessed using the Wilcoxon signed-rank test [26], since the measurement sample was non-normally distributed, as seen from a KolmogorovSmirnov test [50] (the maximum probability value among all the questions was $p=0.010$ for Q5).

Figure 3 shows exemplary signals of the five affective variables acquired by the low-cost EEG device during an experimental session of one subject. For each affective variable, the 
Mouse

(a)

\section{1}

0.5

15

(b)

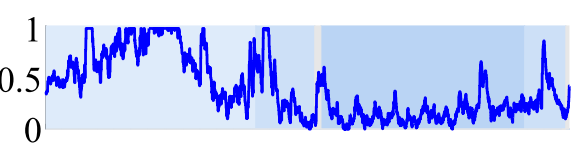

1

(c)

1

0.5

0

(d)

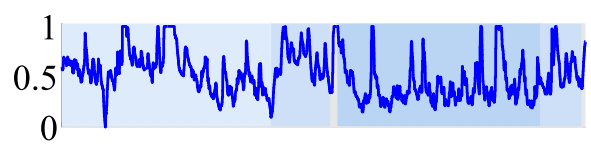

(e)

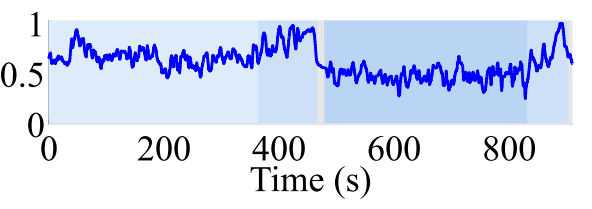

Headset

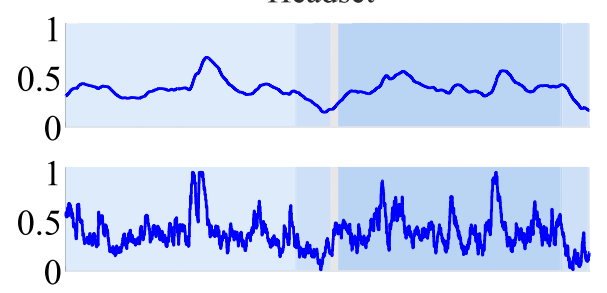

1
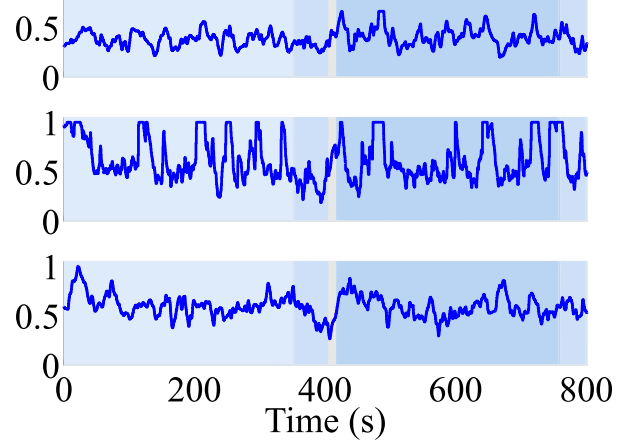

Active navigation Passive navigation Recalling $\quad$ Score viewing

Figure 3: Affective variables acquired during one experimental session in the two different control strategies. (a) Long term Excitement; (b) Short term Excitement; (c) Meditation; (d) Frustration; and (e) Engagement. Graphs in the same column share the same time scale. Different colored areas indicate different trial phases. On the $y$-axis, variables are expressed in arbitrary units, ranging form 0 to 1 , as provided by the EPOC headset

values acquired during the navigation phases were averaged to obtain mean values for the four experimental conditions $(\mathrm{AH}$, $\mathrm{AM}, \mathrm{PH}$, and PM). Both the memory score and the five affective variable values were considered as dependent variables in a two-way repeated measurement ANOVA, with the navigation modality and the interface as the independent factors.

For all the tests, the statistical significance was set at $p=$ 0.05 .

\section{Results}

\subsection{The spatial memory training system was perceived as en- gaging and entertaining}

Participants found the whole activity as interesting and fun, even though not easy. All the collected survey answers were found to be different from chance, except for question Q5 (Q3: $\chi^{2}(4)=55.500, p<0.001 ; \mathrm{Q} 4: \chi^{2}(4)=39.833, p<0.001$; Q5: $\chi^{2}(4)=8.667, p=0.070 ;$ Q6: $\chi^{2}(4)=82.667, p<0.001$; Q7: $\chi^{2}(4)=62.833, p<0.001$; Q8: $\chi^{2}(4)=11.000, p=$ 0.027; and Q9: $\left.\chi^{2}(4)=17.067, p<0.001\right)$.

Figure 4 shows the mean score of the survey (Q3 to Q8) grouped by the three aspects considered (interest, fun, and difficulty). Using the headset was perceived to be more interesting (Q3 vs. Q6: $M_{D}=1.000, Z=-4.506, p<0.001$ ), more fun (Q4 vs. Q7: $M_{D}=1.000, Z=-4.031, p<0.001$ ), and more difficult (Q5 vs. Q8: $M_{D}=1.000, Z=-2.583, p=0.010$ ) than a more traditional mouse. In addition, 46 out of 60 subjects indicated a preference to repeat the trials using the headset.

\subsection{The navigation modality and the interface did not affect memory performance}

Figure 5 reports mean values with $95 \%$ confidential interval of the memory score for the four different conditions considered. Results of the two way ANOVA showed no influence of the navigation modality $(F(1,59)=0.126, p=0.724$, $\left.\eta_{p}^{2}=0.002\right)$, or the interface $(F(1,59)=0.066, p=0.799$, $\left.\eta_{p}^{2}=0.001\right)$ on the memory performance of the involved cohort.

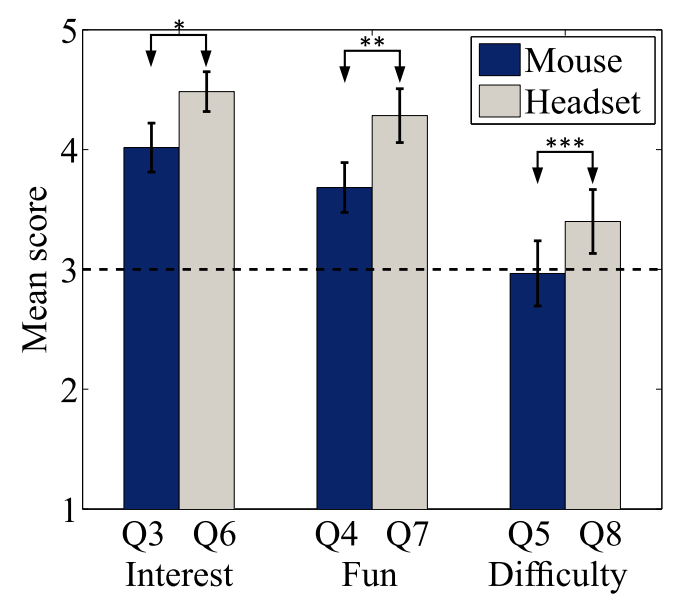

Figure 4: Mean survey scores grouped by interest, fun, and difficulty. The dashed horizontal line indicates the neutral level. Error bars represent $95 \%$ confidence interval. Significant differences are marked with stars: $p\left(^{*}\right)<0.001$, $p\left({ }^{* *}\right)<0.001, p\left(^{* * *}\right)=0.010$. 


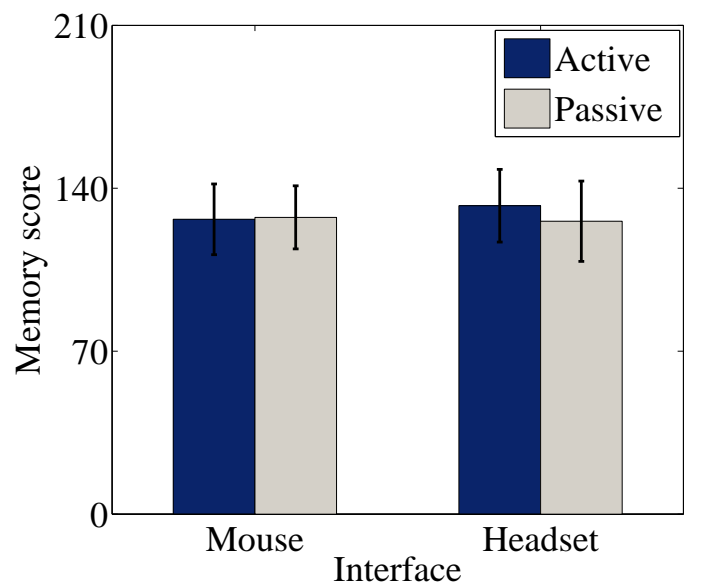

Figure 5: Mean values of memory scores grouped by different interfaces. Error bars represent $95 \%$ confidence interval.

\subsection{The headset was able to detect differences in the affective variables of participants}

Results of the repeated measurement ANOVA showed that the Engagement value provided by the EEG device was higher during active navigation with respect to passive navigation $\left(F(1,59)=24.747, p<0.001, \eta_{p}^{2}=0.296\right)$. In addition, we found a significant effect of the interaction between navigation modalities (A or P) and interface ( $\mathrm{M}$ or $\mathrm{H})$ for the Engagement value $\left(F(1,59)=7.562, p=0.008, \eta_{p}^{2}=0.114\right)$. Specifically, the Engagement was found to be higher when using the mouse with respect to the headset during active navigation, while no difference between the two interfaces was noted during passive navigation, as evidenced in Figure 6-(a).

The interface was found to have a primary influence on the Frustration value $\left(F(1,59)=9.716, p=0.003, \eta_{p}^{2}=0.141\right)$. In particular, Frustration was higher when using the headset than the mouse, with no effect of the navigation modality, as shown in Figure 6-(b). No significant differences were observed on the remaining affective variables (Long term Excitement, Short term Excitement and Meditation) among the different experimental conditions.

\section{Discussion}

In this paper, we presented a novel spatial memory rehabilitation system, envisioned for FAS patients, which was designed around a virtual environment that is part of a citizen science project. The rationale for including scientific content in spatial memory training is grounded in the opportunity of leveraging untapped drivers associated with citizen science toward an engaging, interesting, and effective rehabilitation approach. These motivational drivers, in turn, are expected to compensate for the scarce adherence to rehabilitation regimens that is often seen in FAS patients suffering from severe intellectual disability [21]. At the same time, including citizen science elements in intellectual rehabilitation could offer a new means for increasing scientific literacy and self-esteem in patients, whose learning capacity is unfortunately challenged by attention deficits $[51,38]$.

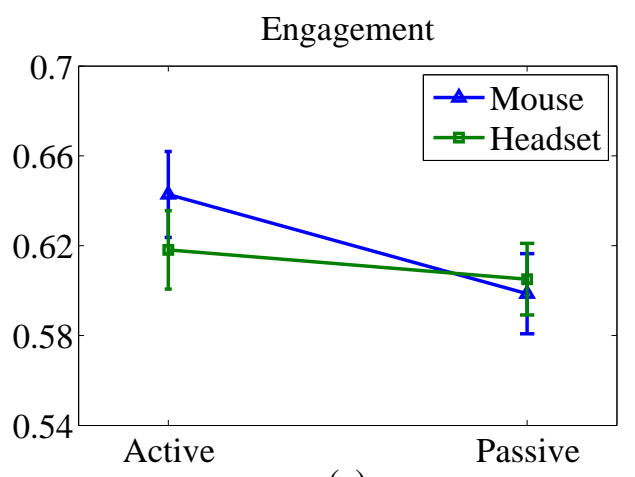

(a)

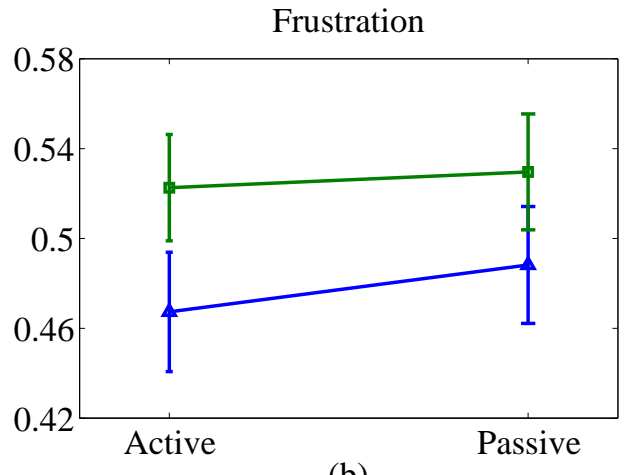

(b)

Figure 6: Mean values of Engagement and Frustration for the four different experimental conditions. Error bars represent 95\% confidence interval. (a) Engagement is significantly higher for active navigation than passive, and there is an effect of the interaction of navigation modality and interface. (b) Frustration is significantly higher using the headset and no interaction effect is noted.

To further enhance the effectiveness of the training system, we explored the possibility of integrating a portable low-cost EEG device for human-computer interactions, which could aid also in affective state recognition.

Our results demonstrate that the training system is perceived as interesting and entertaining by a group of healthy adult subjects, in agreement with our expectations based on previous work on the integration of citizen science activities and science learning in physical rehabilitation $[17,42,60]$. While the subjects did not execute the task on a voluntary basis as the participants of the Brooklyn Atlantis project [44], they were informed of the overall scientific objectives of the project and of the value of the contribution to authentic research in environmental science. Thus, it is tenable to hypothesize that both intrinsic and extrinsic motivations associated with individual interest in the nature of the project and in contributing to research $[57,73,65]$ could be determining factors of the subject perception of the training system. This finding encourages future testing on FAS patients, for whom the interest in the content and the fun in the participation could represent valid predictors of their adherence to rehabilitation treatments $[64,75]$.

Participants rated the headset interface more engaging and entertaining, even if more difficult, than a traditional computer 
mouse. When extending the study to FAS patients, the headset could aid in the successful execution of the spatial memory tasks, whereby subjects could benefit from the intuitiveness of head movements as compared to using a computer mouse. Specifically, natural execution of head movements in a decision making process could strengthen memory performance by reinforcing the distinctiveness of the memory physical substrate in the cerebral circuits [85].

In contrast with our expectations, we did not observe a variation of the memory performance as a function of the navigation modality, active versus passive. Rose et al. [71] reported that active navigation in a virtual environment with respect to passive memorization induced both impaired and non-impaired subjects to perform better in localizing encountered spatial layouts. Conversely, they reported an opposite trend with respect to localizing specific objects in the virtual reality environment, highlighting the complexity of spatial memory assessment [61]. Rose et al. [71] proposed that active navigation benefited the memorization of spatial layouts, but it distracted subjects from memorizing objects that were not key to the navigation. The virtual environmental space [35] presented in our experiment is the reproduction of a real urban canal, whose images cannot be readily classified as spatial layouts or objects as in [71]. Perhaps, such a difference in our experimental design with respect to [71] could be an explaining factor of the lack of an effect of active versus passive navigation in memory performance.

Another possible explanation could be sought in the experimental protocol considered in our study. In [71], subjects in the passive group were asked to watch a video of the navigation performed by a subject of the active group. In our protocol we included the possibility for every subject to freely explore the environment during both active and passive navigation modality, to augment the process of spatial learning, in which a subject should move from an egocentric to an exocentric perception [24]. Thus, in our experimental protocol, active and passive navigation only differed in the additional task of selecting a direction of navigation, as a potential scientific task of the citizen science project.

An additional explanation could involve the typology of the memory test designed, where the location of specific scenes on the map is used to link memory training and science learning. However, spatial knowledge is a multi-dimensional construct [27], which may require more detailed measures than those adopted in this study. For example, future studies should seek to assess the efficacy of the approach through the evaluation of its transferability [7], which is associated with the subject improvement in executing tasks differing from those on which he/she has been trained.

For the group of healthy subjects enrolled in our study, we did not observe an effect of the interface on memory test performance. Translating the study to FAS patients, we would expect that a natural user interface such as the headset could compensate for attention deficit and, consequently, enhance the spatial memory process. This hypothesis rests upon a previous study [82], which demonstrated that patients with attention deficit could exhibit a significantly larger amount of head and body movements during intellectual rehabilitation, increasing their likelihood of being distracted. We would anticipate that linking head movements to the interface could increase their level of attention by offering real-time feedback on head movements. Also, expanding the study to a clinical setting will require a simplification of the task, which was designed for healthy subjects. Considering a simplified version of the test for healthy subjects could cause ceiling effects, which were not observed in our study where the total mean value of the memory score was approximately $43 \%$ of the maximum.

Two of the five affective variables estimated by the Emotiv headset showed a dependence on the experimental condition. Specifically, a higher Engagement value was measured during active navigation. In the field of EEG analysis, such a variable is calculated as the ratio between spectral density of beta waves $(13-30 \mathrm{~Hz})$ and the sum of spectral densities of alpha (7$13 \mathrm{~Hz}$ ) and theta $(4-7 \mathrm{~Hz})$ ones [63]. Correlation between spectral density of those bands and the subjects feelings has been extensively studied over the last decade $[36,46]$. It is generally understood that beta wave density increases with attentiveness and focus, while alpha waves, instead with relaxation, and theta waves with dreaminess and imagination [6]. As a result, Engagement measurement is considered to reflect alertness and conscious direction of attention toward a task [41]. The selection of a particular direction to move and reach a goal could be considered as a decision making process. Consequently, the level of attention during active navigation should increase.

In this perspective, the effect of the interaction between navigation modality and interface represents a particularly interesting result. The increase in concentration due to the additional mental task of navigating the environment seems clear when using the mouse, however it is not evident when using the headset. This suggests that the decision making process of navigating the virtual environment using a computer mouse is more demanding than with the headset, in terms of attention. The adoption of a natural user interface, like the headset, for human-computer interaction process, seems to reduce the level of attention associated with it. However, a difference in the Engagement value between the two interfaces was not found during passive navigation, indicating that the natural user interface might help in coping with the additional decision process; an effect hampered by passive navigation. This preliminary evidence calls for future experimental studies involving higher level of granularity in the subjects' activity to elucidate the link between the process of decision making and the Engagement value measured by the headset.

The Frustration variable was not found to be influenced by the navigation modality, but it was significantly higher when using the headset rather than the mouse. Frustration represents a complex feeling, arising when attempts to achieve a goal are thwarted [68]. According to literature [67, 74], it is often elicited while practicing novel and/or unpredictable control strategies. The result we obtained, is consistent with the survey outcome, where the headset interface was described as more difficult by the subjects. The higher difficulty experienced by the subjects during trials performed with the headset likely produced a feeling of frustration, which was detected by the low-cost EEG device. 
Although the headset was perceived as more entertaining by the subjects, no difference among the four experimental conditions was found for either Long term Excitement or Short term Excitement. These variables are defined as an experience of awareness of positive physiological arousal [41]. Perhaps, the association of the activity with an entertaining experience by subjects should be related with a more complex emotional construct, not simply addressable as the awareness of arousal. Similarly, no difference was observed for the Meditation variable as a function of the navigation modality and the interface. Such a variable is supposed to express a relaxed state of mind [41], which was not expected to vary between conditions that were all designed to maintain the subject alert for executing the spatial memory test.

Our findings suggest that the inclusion of a citizen science context, together with the use of a portable low-cost EEG device for human-computer interaction, could offer a viable means to design engaging, entertaining, and effective intellectual rehabilitation exercises. In particular, our results open the way for a future exploration of two new possibilities of intellectual rehabilitation of FAS patients: (i) integrating a low-cost EEG-based affective bio-feedback control and (ii) providing clinicians with an array of novel indices of adherence to the therapy based on the measurement of affective variables. The patient-specific bio-feedback control will afford the possibility of adjusting the cognitive load [52] during the execution of the spatial memory task based on real-time measurement of the Engagement and Frustration variables, potentially enhancing the effectiveness of the intellectual rehabilitation activity. These activities can, in turn, be used to help patients improving their academic performance, gain familiarity with science concepts, and contribute to authentic research projects.

\section{Conflict of Interest}

The authors declare no conflict of interest.

\section{References}

\section{References}

[1] Abel, E. L., Sokol, R. J., 1987. Incidence of fetal alcohol syndrome and economic impact of fas-related anomalies. Drug and Alcohol Dependence 19 (1), 51-70.

[2] Al-Hudhud, G., 2014. Affective command-based control system integrating brain signals in commands control systems. Computers in Human Behavior 30, 535-541.

[3] Alloway, T. P., 2006. How does working memory work in the classroom? Educational Research and Reviews 1 (4), 134-139.

[4] Alloway, T. P., 2007. Automated Working: Memory Assessment: Manual. Pearson.

[5] Alloway, T. P., Bibile, V., Lau, G., 2013. Computerized working memory training: Can it lead to gains in cognitive skills in students? Computers in Human Behavior 29 (3), 632-638.

[6] Andujar, M., Gilbert, J. E., 2013. Let's learn!: enhancing user's engagement levels through passive brain-computer interfaces. In: CHI' 13 Extended Abstracts on Human Factors in Computing Systems. ACM, pp. 703-708.

[7] Baldwin, T. T., Ford, J. K., 1994. Transfer of training: A review and directions for future research. The training and development sourcebook 180.
[8] Barnes, D. E., Walker, D. W., 1981. Prenatal ethanol exposure permanently reduces the number of pyramidal neurons in rat hippocampus. Developmental Brain Research 1 (3), 333-340.

[9] Barnett, R., Maruff, P., Vance, A., Luk, E., Costin, J., Wood, C., Pantelis, C., 2001. Abnormal executive function in attention deficit hyperactivity disorder: The effect of stimulant medication and age on spatial working memory. Psychological Medicine 31 (06), 1107-1115.

[10] Bartle, D., Rossoff, S., Whittaker, D., Gooch, B., Kerns, K., MacSween, J., 2010. Cognitive games as therapy for children with FAS. In: ACM SIGGRAPH 2010 Posters. ACM, p. 48.

[11] Breathnach, C., 1980. The hippocampus as a cognitive map. Philosophical Studies 27, 263-267.

[12] Breslow, L., Gartenberg, D., McCurry, J. M., Trafton, J. G., et al., 2014. Dynamic operator overload: A model for predicting workload during supervisory control. IEEE Transactions on Human-Machine Systems 44 (1), $30-40$.

[13] Burgers, C., Eden, A., van Engelenburg, M. D., Buningh, S., 2015. How feedback boosts motivation and play in a brain-training game. Computers in Human Behavior 48, 94-103.

[14] Burgess, D. M., Streissguth, A. P., 1992. Fetal alcohol syndrome and fetal alcohol effects: Principles for educators. Phi Delta Kappan, 24-30.

[15] Butnik, S. M., 2005. Neurofeedback in adolescents and adults with attention deficit hyperactivity disorder. Journal of Clinical Psychology 61 (5), 621-625.

[16] Cappa, F., Laut, J., Nov, O., Giustiniano, L., Porfiri, M., 2016. Activating social strategies: Face-to-face interaction in technology-mediated citizen science. Journal of Environmental Management 182, 374-384.

[17] Cappa, P., Clerico, A., Nov, O., Porfiri, M., 2013. Can force feedback and science learning enhance the effectiveness of neuro-rehabilitation? An experimental study on using a low-cost 3D joystick and a virtual visit to a zoo. PLoS ONE 8 (12), e83945.

[18] Clarren, S. K., Smith, D. W., 1978. The fetal alcohol syndrome. The New England Journal of Medicine.

[19] Dennett, H. W., McKone, E., Tavashmi, R., Hall, A., Pidcock, M., Edwards, M., Duchaine, B., 2012. The cambridge car memory test: A task matched in format to the cambridge face memory test, with norms, reliability, sex differences, dissociations from face memory, and expertise effects. Behavior Research Methods 44 (2), 587-605.

[20] Dickinson, J. L., Zuckerberg, B., Bonter, D. N., 2010. Citizen science as an ecological research tool: Challenges and benefits. Annual Review of Ecology, Evolution, and Systematics 41, 149-172.

[21] Dovis, S., Van der Oord, S., Wiers, R. W., Prins, P. J., 2015. Improving executive functioning in children with ADHD: Training multiple executive functions within the context of a computer game. A randomized double-blind placebo controlled trial. PLoS ONE 10 (4), e0121651.

[22] Duvinage, M., Castermans, T., Petieau, M., Hoellinger, T., Cheron, G., Dutoit, T., 2013. Performance of the Emotiv Epoc headset for p300-based applications. Biomedical Engineering Online 12, 56.

[23] Emotiv, June 2015. Emotiv EPOC. https://emotiv . com/epoc.php.

[24] Evans, G. W., 1980. Environmental Cognition. Psychological Bulletin 88 (2), 259

[25] Gianoulakis, C., 1990. Rats exposed prenatally to alcohol exhibit impairment in spatial navigation test. Behavioural Brain Research 36 (3), 217 228.

[26] Gibbons, J. D., Chakraborti, S., 2011. Nonparametric Statistical Inference. Springer.

[27] Golledge, R. G., 1999. Human wayfinding and cognitive maps. Wayfinding behavior: cognitive mapping and other spatial processes, 5-45.

[28] Green, C., Mihic, A., Nikkel, S., Stade, B., Rasmussen, C., Munoz, D., Reynolds, J., 2009. Executive function deficits in children with fetal alcohol spectrum disorders (FASD) measured using the cambridge neuropsychological tests automated battery (CANTAB). Journal of Child Psychology and Psychiatry 50 (6), 688-697.

[29] Haklay, M., 2013. Citizen science and volunteered geographic information: Overview and typology of participation. In: Crowdsourcing Geographic Knowledge. Springer, pp. 105-122.

[30] Henry, L. A., MacLean, M., 2002. Working memory performance in children with and without intellectual disabilities. Journal Information 107 (6).

[31] Jacobs, W. J., Laurance, H. E., Thomas, K. G., 1997. Place learning in virtual space I: Acquisition, overshadowing, and transfer. Learning and 
Motivation 28 (4), 521-541.

[32] Jaeggi, S. M., Buschkuehl, M., Jonides, J., Perrig, W. J., 2008. Improving fluid intelligence with training on working memory. Proceedings of the National Academy of Sciences 105 (19), 6829-6833.

[33] Jasper, H. H., 1958. The ten twenty electrode system of the international federation. Electroencephalography and Clinical Neurophysiology 10, 371-375.

[34] Kim, M.-K., Kim, M., Oh, E., Kim, S.-P., 2013. A review on the computational methods for emotional state estimation from the human EEG. Computational and Mathematical Methods in Medicine 2013.

[35] Kitchin, R., Blades, M., 2002. The cognition of geographic space. Vol. 4. Ib Tauris.

[36] Klimesch, W., 1999. Eeg alpha and theta oscillations reflect cognitive and memory performance: a review and analysis. Brain Research Reviews 29 (2), 169-195.

[37] Klingberg, T., Forssberg, H., Westerberg, H., 2002. Increased brain activity in frontal and parietal cortex underlies the development of visuospatial working memory capacity during childhood. Journal of Cognitive Neuroscience 14 (1), 1-10.

[38] Kodituwakku, P. W., 2009. Neurocognitive profile in children with fetal alcohol spectrum disorders. Developmental Disabilities Research Reviews 15 (3), 218.

[39] Kodituwakku, P. W., Kodituwakku, E. L., 2011. From research to practice: An integrative framework for the development of interventions for children with fetal alcohol spectrum disorders. Neuropsychology Review 21 (2), 204-223.

[40] Kropotov, Y. D., Ponomarev, V., et al., 2001. EEG-biocontrol method in treating the attention deficit hyperactivity disorder in children. Human Physiology 27 (4), 496-504.

[41] Lang, M., 2012. Investigating the Emotiv Epoc for cognitive control in limited training time. Honours Report, University of Canterbury, 8.

[42] Laut, J., Cappa, F., Nov, O., Porfiri, M., 2015. Increasing patient engagement in rehabilitation exercises using computer-based citizen science. PLoS ONE 10 (3), e0117013.

[43] Laut, J., Cappa, F., Nov, O., Porfiri, M., 2016. Increasing citizen science contribution using a virtual peer. Journal of the Association for Information Science and Technology.

[44] Laut, J., Henry, E., Nov, O., Porfiri, M., 2014. Development of a mechatronics-based citizen science platform for aquatic environmental monitoring. IEEE/ASME Transactions on Mechatronics 19 (5), 15411551 .

[45] Loomes, C., Rasmussen, C., Pei, J., Manji, S., Andrew, G., 2008. The effect of rehearsal training on working memory span of children with fetal alcohol spectrum disorder. Research in Developmental Disabilities 29 (2), 113-124.

[46] Lotte, F., Congedo, M., Lécuyer, A., Lamarche, F., Arnaldi, B., 2007. A review of classification algorithms for eeg-based brain-computer interfaces. Journal of Neural Engineering 4 (2), R1.

[47] Maguire, E., Frith, C., Burgess, N., Donnett, J., O’Keefe, J., 1998. Knowing where things are: Parahippocampal involvement in encoding object locations in virtual large-scale space. Journal of Cognitive Neuroscience 10 (1), 61-76.

[48] Mandryk, R. L., Dielschneider, S., Kalyn, M. R., Bertram, C. P., Gaetz, M., Doucette, A., Taylor, B. A., Orr, A. P., Keiver, K., 2013. Games as neurofeedback training for children with FASD. In: Proceedings of the 12th International Conference on Interaction Design and Children. ACM, pp. 165-172.

[49] Maps, G., March 2015. Brooklyn, NY.Map. https://www.google. $\mathrm{com} / \mathrm{maps} / @ 40.6753639,-73.9917184,3756 \mathrm{a}, 20 \mathrm{y}, 114.75 \mathrm{~h} /$ data $=$ ! $3 \mathrm{~m} 1$ ! $1 \mathrm{e} 3$.

[50] Massey Jr, F. J., 1951. The Kolmogorov-Smirnov test for goodness of fit. Journal of the American Statistical Association 46 (253), 68-78.

[51] Mattson, S. N., Crocker, N., Nguyen, T. T., 2011. Fetal alcohol spectrum disorders: Neuropsychological and behavioral features. Neuropsychology Review 21 (2), 81-101.

[52] Mayer, R. E., Moreno, R., 2003. Nine ways to reduce cognitive load in multimedia learning. Educational Psychologist 38 (1), 43-52.

[53] Mihajlovic, V., Grundlehner, B., Vullers, R., Penders, J., 2015. Wearable, wireless eeg solutions in daily life applications: What are we missing? IEEE Journal of Biomedical and Health Informatics 19 (1), 6-21.

[54] Moore, M. M., Dua, U., 2004. A galvanic skin response interface for peo- ple with severe motor disabilities. In: ACM SIGACCESS Accessibility and Computing. No. 77-78. ACM, pp. 48-54.

[55] Morris, R. G., 1981. Spatial localization does not require the presence of local cues. Learning and Motivation 12 (2), 239-260.

[56] NI, June 2015. LabVIEW Emotiv toolkit. https://decibel.ni.com/ content/docs/DOC-18059.

[57] Nov, O., Arazy, O., Anderson, D., 2014. Scientists@ home: What drives the quantity and quality of online citizen science participation? PLoS ONE 9 (4), e90375.

[58] NYU, June 2015. Brooklyn Atlantis. http://www . brooklynatlantis.poly.edu/.

[59] Olton, D. S., Becker, J. T., Handelmann, G. E., 1979. Hippocampus, space, and memory. Behavioral and Brain Sciences 2 (03), 313-322.

[60] Palermo, E., Laut, J., Nov, O., Cappa, P., Porfiri, M., 2017. A natural user interface to integrate citizen science and physical exercise. PloS ONE 12 (2), e0172587.

[61] Péruch, P., Vercher, J.-L., Gauthier, G. M., 1995. Acquisition of spatial knowledge through visual exploration of simulated environments. Ecological Psychology 7 (1), 1-20.

[62] Pett, M. A., 1997. Nonparametric statistics in health care research: Statistics for small samples and unusual distributions. Sage.

[63] Pope, A. T., Bogart, E. H., Bartolome, D. S., 1995. Biocybernetic system evaluates indices of operator engagement in automated task. Biological Psychology 40 (1), 187-195.

[64] Prins, P. J., Dovis, S., Ponsioen, A., Ten Brink, E., Van der Oord, S., 2011. Does computerized working memory training with game elements enhance motivation and training efficacy in children with ADHD? $\mathrm{Cy}$ berpsychology, Behavior, and Social Networking 14 (3), 115-122.

[65] Raddick, M. J., Bracey, G., Gay, P. L., Lintott, C. J., Murray, P., Schawinski, K., Szalay, A. S., Vandenberg, J., 2010. Galaxy zoo: Exploring the motivations of citizen science volunteers. Astronomy Education Review 9 (1), 010103.

[66] Reid, I., Wright, N., Whalley, L., 1995. Arenamaze a virtual watermaze for humans. In: Neuroscience Abstracts. Vol. 21. p. 1446

[67] Reuderink, B., Mühl, C., Poel, M., 2013. Valence, arousal and dominance in the eeg during game play. International Journal of Autonomous and Adaptive Communications Systems 6 (1), 45-62.

[68] Reynolds, C. J., 2001. The sensing and measurement of frustration with computers. Ph.D. thesis, Massachusetts Institute of Technology.

[69] Riley, E. P., McGee, C. L., Sowell, E. R., 2004. Teratogenic effects of alcohol: A decade of brain imaging. In: American Journal of Medical Genetics Part C: Seminars in Medical Genetics. Vol. 127. Wiley Online Library, pp. 35-41.

[70] Rizzo, A. A., Buckwalter, J. G., Bowerly, T., Van Der Zaag, C., Humphrey, L., Neumann, U., Chua, C., Kyriakakis, C., Van Rooyen, A., Sisemore, D., 2000. The virtual classroom: A virtual reality environment for the assessment and rehabilitation of attention deficits. CyberPsychology \& Behavior 3 (3), 483-499.

[71] Rose, D., Brooks, B., Attree, E., Parslow, D., Leadbetter, A., McNeil, J., Jayawardena, S., Greenwood, R., Potter, J., 1999. A preliminary investigation into the use of virtual environments in memory retraining after vascular brain injury: Indications for future strategy? Disability \& Rehabilitation 21 (12), 548-554.

[72] Rose, F. D., Brooks, B. M., Rizzo, A. A., 2005. Virtual reality in brain damage rehabilitation: Review. CyberPsychology \& Behavior 8 (3), 241262.

[73] Rotman, D., Preece, J., Hammock, J., Procita, K., Hansen, D., Parr, C., Lewis, D., Jacobs, D., 2012. Dynamic changes in motivation in collaborative citizen-science projects. In: Proceedings of the ACM 2012 conference on Computer Supported Cooperative Work. ACM, pp. 217-226.

[74] Scheirer, J., Fernandez, R., Klein, J., Picard, R. W., 2002. Frustrating the user on purpose: a step toward building an affective computer. Interacting with Computers 14 (2), 93-118.

[75] Shaw, R., Grayson, A., Lewis, V., 2005. Inhibition, ADHD, and computer games: The inhibitory performance of children with ADHD on computerized tasks and games. Journal of Attention Disorders 8 (4), 160-168.

[76] Shelton, C. E., Luo, Y., Shen, Y., 2013. Measurement and analysis of braille stimulus to brain using an EEG: A preliminary study. In: IEEE 3rd Annual International Conference on Cyber Technology in Automation, Control and Intelligent Systems (CYBER), 2013. IEEE, pp. 385-389.

[77] Silvertown, J., 2009. A new dawn for citizen science. Trends in Ecology 
\& Evolution 24 (9), 467-471.

[78] Skelton, R. W., Ross, S. P., Nerad, L., Livingstone, S. A., 2006. Human spatial navigation deficits after traumatic brain injury shown in the arena maze, a virtual Morris water maze. Brain Injury 20 (2), 189-203.

[79] Smith, S. P., Stibric, M., Smithson, D., 2013. Exploring the effectiveness of commercial and custom-built games for cognitive training. Computers in Human Behavior 29 (6), 2388-2393.

[80] Sourina, O., Liu, Y., 2014. EEG-enabled affective human-computer interfaces. In: Universal Access in Human-Computer Interaction. Design and Development Methods for Universal Access. Springer, pp. 536-547.

[81] Swangnetr, M., Kaber, D. B., 2013. Emotional state classification in patient-robot interaction using wavelet analysis and statistics-based feature selection. IEEE Transactions on Human-Machine Systems 43 (1), 63-75.

[82] Teicher, M. H., Ito, Y., Glod, C. A., Barber, N. I., 1996. Objective measurement of hyperactivity and attentional problems in ADHD. Journal of the American Academy of Child \& Adolescent Psychiatry 35 (3), 334 342 .

[83] Thackray, H. M., Tifft, C., 2001. Fetal alcohol syndrome. Pediatrics in Review 22 (2), 47-55.

[84] VandenBos, G. R., 2007. APA Dictionary of Psychology. American Psychological Association.

[85] Voss, J. L., Gonsalves, B. D., Federmeier, K. D., Tranel, D., Cohen, N. J., 2011. Hippocampal brain-network coordination during volitional exploratory behavior enhances learning. Nature Neuroscience 14, 115120.

[86] Wang, Q., Sourina, O., Nguyen, M. K., 2010. EEG-based serious games design for medical applications. In: International Conference on Cyberworlds (CW). IEEE, pp. 270-276.

[87] Zhang, J., Yin, Z., Wang, R., 2015. Recognition of mental workload levels under complex human-machine collaboration by using physiological features and adaptive support vector machines. IEEE Transactions on Human-Machine Systems 45 (2), 200-214. 